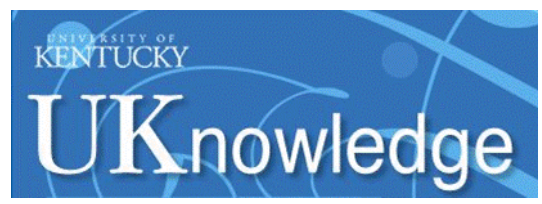

University of Kentucky

UKnowledge

$7-2010$

\title{
White Matter Diffusion Alterations in Normal Women at Risk of Alzheimer's Disease
}

\author{
Charles D. Smith \\ University of Kentucky, csmith@mri.uky.edu \\ Himachandra Chebrolu \\ University of Kentucky \\ Anders H. Andersen \\ University of Kentucky, anders@mri.uky.edu \\ David A. Powell \\ University of Kentucky \\ Mark A. Lovell \\ University of Kentucky, malove2@pop.uky.edu
}

See next page for additional authors

Follow this and additional works at: https://uknowledge.uky.edu/neurology_facpub

Part of the Chemistry Commons, Medical Neurobiology Commons, and the Neurology Commons

Right click to open a feedback form in a new tab to let us know how this document benefits you.

\section{Repository Citation}

Smith, Charles D.; Chebrolu, Himachandra; Andersen, Anders H.; Powell, David A.; Lovell, Mark A.; Xiong, Shuling; and Gold, Brian T., "White Matter Diffusion Alterations in Normal Women at Risk of Alzheimer's Disease" (2010). Neurology Faculty Publications. 1.

https://uknowledge.uky.edu/neurology_facpub/1

This Article is brought to you for free and open access by the Neurology at UKnowledge. It has been accepted for inclusion in Neurology Faculty Publications by an authorized administrator of UKnowledge. For more information, please contact UKnowledge@lsv.uky.edu. 


\section{White Matter Diffusion Alterations in Normal Women at Risk of Alzheimer's Disease}

Digital Object Identifier (DOI)

http://dx.doi.org/10.1016/j.neurobiolaging.2008.08.006

Notes/Citation Information

Published in Neurobiology of Aging, v. 31, issue 7, p. 1122-1131.

(c) 2008 Elsevier Inc. All rights reserved.

This manuscript version is made available under the CC-BY-NC-ND 4.0 license

http://creativecommons.org/licenses/by-nc-nd/4.0/

Authors

Charles D. Smith, Himachandra Chebrolu, Anders H. Andersen, David A. Powell, Mark A. Lovell, Shuling Xiong, and Brian T. Gold 
(C) 2008 Elsevier Inc. All rights reserved.

This manuscript version is made available under the CC-BY-NC-ND 4.0 license

http://creativecommons.org/licenses/by-nc-

nd/4.0/ 


\title{
White matter diffusion alterations in normal women at risk of Alzheimer's disease
}

\author{
Charles D. Smith ${ }^{a, b, c},{ }^{*}$, Himachandra Chebrolu ${ }^{b}$, Anders H. Andersen ${ }^{b, c}$, David A. Powellb, \\ Mark A. Lovelld, Shuling Xiong ${ }^{d}$, and Brian T. Gold ${ }^{\mathrm{C}}$ \\ aDepartment of Neurology, University of Kentucky, Lexington, KY 40536, United States \\ bMagnetic Resonance Imaging and Spectroscopy Center, University of Kentucky, Lexington, KY \\ 40536, United States \\ 'Department of Anatomy and Neurobiology, University of Kentucky, Lexington, KY 40536, United \\ States \\ dDepartment of Chemistry, University of Kentucky, Lexington, KY 40536, United States
}

\begin{abstract}
Increased white matter mean diffusivity and decreased fractional anisotropy (FA) has been observed in subjects diagnosed with mild cognitive impairment (MCI) and Alzheimer's disease (AD). We sought to determine whether similar alterations of white matter occur in normal individuals at risk of AD. Diffusion tensor images were acquired in 42 cognitively normal right-handed women with both a family history of dementia and at least one apolipoprotein E4 allele. These were compared with images from 23 normal women without either AD risk factor. Group analyses were performed using tract-based spatial statistics. Reduced FA was observed in the fronto-occipital and inferior temporal fasciculi (particularly posteriorly), the splenium of the corpus callosum, subcallosal white matter and the cingulum bundle. These findings demonstrate that specific white matter pathways are altered in normal women at increased risk of $\mathrm{AD}$ years before the expected onset of cognitive symptoms.
\end{abstract}

\section{Keywords}

Diffusion tensor; Fractional anisotropy; Magnetic resonance imaging; Alzheimer's disease; Apolipoprotein E

\section{Introduction}

There is now strong evidence that significant Alzheimer's neuropathology is present years before any symptoms of mild cognitive impairment (MCI) or Alzheimer's disease (AD) appear (Braak and Braak, 1997; Bennett et al., 2006). The underlying theory of this study is that the first appearance of cognitive symptoms due to $\mathrm{AD}$ pathology, e.g., amnestic MCI, represents

(C) 2008 Elsevier Inc. All rights reserved

*Corresponding author at: Magnetic Resonance Imaging and Spectroscopy Center, Room 62, University of Kentucky Medical Center, 800 Rose Street, Lexington, KY 40536-0098, United States. Tel.: +1 859323 1113; fax: +1 859323 1068. E-mail address: csmith@mri.uky.edu (C.D. Smith)..

Conflict of interest None.

Appendix A. Supplementary data Supplementary data associated with this article can be found, in the online version, at doi:10.1016/ j. neurobiolaging.2008.08.006. 
early failure of brain compensatory mechanisms and cerebral reserve, a critical point in the further evolution of neuronal injury produced by that pathology (Smith, 2007). We assume that $\mathrm{AD}$ neuropathology progresses with time. But even if a treatment could theoretically slow or arrest MCI/AD, other processes of aging and vascular injury could operate on this substrate of critical reserve and result in further cognitive decline (Schneider et al., 2007). Therefore, identifying the brain at risk before $\mathrm{MCI}$ is important to the goal of preventing the critical point from ever being reached, by using AD disease-modifying treatments, combined with treatments aimed at the comorbid pathologies and enhancement of brain reserve, before symptoms appear.

Several methodologies have been applied, or have potential to be applied, to asymptomatic persons to assess the brain at $\mathrm{AD}$ risk, including cerebrospinal fluid protein patterning, genetic analysis, resting glucose positron emission tomography, and functional and structural magnetic resonance imaging. Studies of white matter integrity using magnetic resonance diffusion tensor imaging have demonstrated alterations in the frontal, temporal and parietal lobes in subjects with MCI and AD (Medina et al., 2006; Xie et al., 2006; Fellgiebel et al., 2007; Firbank et al., 2007; Huang et al., 2007; Stahl et al., 2007; Sydykova et al., 2007; Teipel et al., 2007; Zhang et al., 2007; Ding et al., 2008; Rose et al., 2008; Yasmin et al., 2008). There is pathological evidence that disruption of white matter in Alzheimer's disease may be an early event (Englund et al., 1988). In line with this data, there is important recent evidence suggesting that normal persons at increased risk for late-onset AD by virtue of an apolipoprotein-E4 (APOE4) allele show reduced fractional anisotropy (FA) in certain brain regions compared to low-risk groups (Nierenberg et al., 2005; Persson et al., 2006a,b). However, these studies have either explored only a few regions with ROI analyses, or used automated techniques known to have limitations related to registration and smoothing (Bookstein, 2001; Smith et al., 2006). In the present study we applied rigorously validated methods for comparing group fractional anisotropy maps across the brain's white matter (Smith et al., 2006) between well-characterized normal subjects who vary in their risk of AD. The main advantages of these methods are improved registration of white matter, the lack of a need to apply an arbitrary smoothing kernel, and a common reference framework (mean FA skeleton). In addition, because the method is voxel-based, arbitrary definitions of region of interest are not required.

\section{Methods}

\subsection{Subjects}

All subjects are participants in a University of Kentucky longitudinal imaging study of normal persons who vary in their risk of AD based on family history of dementia and APOE allele status (Smith et al., 1999, 2002). Inclusion criteria include generally good health without memory complaints, stable medication regimen, right handed female between the age of 40 and 90 years, vision correctable to at least 20/50, and knowledge of family history (FH). Family history was considered positive if one or more first-degree relatives had late-onset progressive dementia. Only right-handed females were recruited because they are part of a larger multimodal imaging study that includes functional magnetic resonance imaging during language-based tasks. Language task-based fMRI is known to demonstrate gender and handedness activation differences.

Exclusions include current medical evaluation or treatment for acute or worsening complaints (e.g., unstable asthma), stroke or other cerebral injury, psychoactive medications except stable doses of serotonin reuptake inhibitors and absence of active depression, significant head injury (operationally loss of consciousness greater than $5 \mathrm{~min}$ ), significant psychiatric history, claustrophobia, metallic implants in the head or neck, pacemakers, major surgery within 3 months, or death of one or both parents before age 65 . 
Participants who are selected for scanning based on risk undergo standardized psychometric testing which includes the Mini Mental State Examination (MMSE), Trails A (TA) and B (TB), FAS Letter Fluency (LF), Wechsler Adult Intelligence Scale (WAIS) Block Design (Block Des) and Vocabulary (Vocab), 15-item Boston Naming Test (BNT), Wechsler Memory Scale (WMS) Logical Memory I (LM Imm) and II (LM Del), Visual Reproduction immediate and delayed (VR and VR Del), Digit Span (DS), Spatial Span (SS), Selective Reminding Test Immediate and Delayed (SRT \& SRT Del), and Beck depression inventory (Beck).

Abbreviations are given for reference to Table 1B. These tests are given to exclude persons with cognitive impairment and to document equivalent normal cognitive test performance between risk groups. All subjects had an APOE allele determination. APOE alleles were determined using polymerase chain reaction (PCR) amplification followed by enzymatic digestion and separation of DNA fragments by agarose gel electrophoresis. Informed consent was obtained from each enrollee under an approved University of Kentucky medical institutional review board protocol.

Forty-four high risk (both FH dementia and one or more APOE4 alleles) and 27 low risk (neither FH nor APOE4) participants underwent diffusion tensor magnetic resonance imaging (DT-MRI) between the years 2006 and 2007. One of each risk group were excluded because of poor memory performance on cognitive testing (1.5 S.D. below the mean on Logical Memory II), and an additional one of the high risk and three of the low-risk group were not analyzed because of poor quality MRI scans. None of the included scans demonstrated small or large-vessel infarcts. Consequently there were 42 in the high-risk and 23 in the low-risk group available for DT analysis. Demographic information and testing results are summarized in Table 1. Psychometric scores that sample general mental ability, processing speed, and delayed verbal and nonverbal memory are provided in the table.

Demographic and psychometric scores were compared between risk groups. First age and education were entered as response variables in a standard least-squares analysis with risk group as the independent variable (Table 1A). Age and education were chosen as the most likely to variables to affect psychometric test scores. Because all subjects were right-handed females, gender and handedness were not factors. A standard least-squares model was then developed for the psychometric test scores, with risk group, age, education and risk by age interaction as the independent variables versus the following independent variables: MMSE, Logical Memory immediate and delayed, Block Design, Visual Reproduction immediate and delayed, Boston naming, Vocabulary, Selective Reminding Test immediate and delayed, Trails A and B, Letter Fluency, Spatial Span, Digit Symbol, and Beck inventory score (Table 1B).

To test for potential differences between risk groups related to vascular factors or estrogen replacement that might affect white matter, Chi-Square analysis using Pearson's criterion without correction for multiple comparisons was performed on the following categorical variables (Present/Not Present): current smoking, past smoking, current estrogen replacement, past estrogen replacement, hypercholesterolemia, hypertension and diabetes. There were three subjects with stable diabetes in the study, one in the low-and two in the high-AD risk group. None of the $p$-values in these comparisons was less than $p=0.16$, demonstrating that the frequencies of these factors were the same between risk groups. More detailed data is presented in a supplementary table on the journal web site (http://www.elsevier.com).

\subsection{MRI protocol}

Scanning was performed on a Siemens Trio 3T instrument. Whole-brain (40-slice) diffusion tensor images were acquired with 12 encoding directions with 3 averages each $\left(\mathrm{B}_{0}\right.$ images were averaged 6 times; TR $=14600 \mathrm{~ms}$, TE $=96 \mathrm{~ms}$; resolution $1.8 \mathrm{~mm} \times 1.8 \mathrm{~mm} \times 3.0 \mathrm{~mm}$ ) using a fluid attenuated inversion recovery (FLAIR) double-refocused spin echo sequence. 


\subsection{Image processing and statistics}

Voxelwise statistical analysis of the FA data was carried out using TBSS (Tract-Based Spatial Statistics (Smith et al., 2006)), part of the publicly available image processing software FSL 4.0 (http://www.fmrib.ox.ac.uk/fsl/tbss/index.html). TBSS was used because its algorithms for alignment of FA images from multiple subjects into a common space have been extensively tested and validated (Smith et al., 2006). In addition, the technique does not require spatial smoothing of images, which can produce different results based upon the kernel size (Jones et al., 2005). All of the processing modules referred to below by their acronym are available for download at the FSL web site. First, FA images were created by fitting a tensor model to the raw diffusion data using FSL's Diffusion Toolbox, which consists of a series of tools for lowlevel diffusion parameter reconstruction and probabilistic tractography.

In order to minimize the extent of warping required for registration to a common target, a subset of 25 subjects was used to select a target image for nonlinear registration. Subjects forming both the whole set and the subset were scanned over the same time interval. The subset was selected in two steps. First, the brain volumes of all subjects were calculated and rank ordered. Second, age-volume means within the interquartile range were arranged in chronological order, and the 25 subjects closest in age to the median were selected. From the 25 subjects, the optimum target image was found.

The subset subjects' FA images were aligned with every other subset subjects' FA images using the nonlinear registration toolkit IRTK (Rueckert et al., 1999)

(http://www.doc.ic.ac.uk/ dr/software). The common target FA image for the nonlinear registration was chosen as the FA image for which the least amount of total nonlinear warping across all subset subjects was necessary for registration to the same space. The least amount of warping was determined by registration of each subject to every other subject, and summarization of each warp field by its mean displacement. The resulting target FA image was then affine registered to MNI space. A single transformation matrix for each of the 65 subjects, combining the nonlinear registration of the subject's FA image to the target FA image and the affine registration of the target FA image to MNI space, was then applied. The individual FA images were resampled at $1 \mathrm{~mm} \times 1 \mathrm{~mm} \times 1 \mathrm{~mm}$ isotropic voxels using sinc interpolation.

Next, a mean FA image from all of the subjects was created and thinned with a FA threshold of 0.2 to create a mean FA skeleton which represents the centers of all tracts common to the group. Each subject's aligned FA data was then projected onto this skeleton and the resulting data analyzed using voxelwise cross-subject statistics. A false discovery rate (FDR) threshold scaled to an alpha of $p=0.05$ was determined via random simulations (using 5000 permutations) to correct for multiple comparisons (implemented as in: http://www.fmrib.ox.ac.uk/fsl/randomise/fdr.html). The FDR was then applied to all voxelbased group comparisons. Centered age was used as a covariate in the analysis, because the risk groups showed an overall difference in age (see Section 3).

\section{Results}

Demographic and testing characteristics of the study groups are given in Table 1. Because no correction was made for the 16 multiple comparisons in Table 1B, we chose to conservatively interpret $p$ between 0.01 and 0.05 as indicating marginal significance, and $p$ less than 0.01 as significant. Low-AD risk subjects were older than high-risk subjects due to the convenience nature of the sample. We speculate that normal working-age high-risk younger subjects may be more motivated to volunteer their time for this study than similarly aged low-risk volunteers because of personal experience with Alzheimer's disease in family members. In previous studies using APOE allele status for risk stratification, the average age was approximately 67 
years (Nierenberg et al., 2005;Persson et al., 2006a,b), compared to approximately 58 years for high-risk subjects in the present study. The time to expected development of MCI or AD is therefore about 9 years greater in the present study. Education level was not different between risk groups. The frequencies of past and present hypertension, hypercholesterolemia, smoking and estrogen replacement were similar between risk groups (Supplemental Table).

Correlation with age was in the expected positive direction for Trails A and B, and in the negative direction for Block Design, Visual Reproduction Immediate and Delayed, Selective Reminding Test Delayed, and Spatial Span. Education effects were observed only marginally on the Beck inventory; the absence of these effects overall is attributed to the similar mean and narrow range of education in the study subjects (Table 1B). The age $\times$ risk interaction was marginally significant for LM immediate interaction, scores decreased with increasing age in the low-risk group but not for the high-risk group, whereas VR immediate scores decreased with increasing age in the high-risk group but not for the low-risk group.

Trails A was the only psychometric test of the 16 performed meeting our threshold $p$-value of $<0.01$ for risk group difference. Trails $\mathrm{A}$ is a visuomotor task combining visuospatial and motor speed components. High-risk subjects were slower on this test than low risk. High-risk subjects were also slower on Trails B, but not significantly. The difference between Trails B and A is thought to produce a variable with reduced dependence on motor speed. This B minus A difference when compared between groups was not significant, suggesting that lower performance on Trails A in high-AD risk subjects was due to decreased motor speed and not visuospatial factors (high risk 39.9 \pm 3.7 S.E.M., low risk 35.7 \pm 5.2 S.E.M. seconds; $p=0.5$ ). As expected, age was positively correlated with B minus A $(p=0.02)$. Spatial span was lower in high-risk subjects but not within our cutoff for group difference (Table 1B). In contrast, there were no differences between risk groups in general mental ability (MMSE, Vocabulary, BNT) or in memory measures (LM delayed, or in the more sensitive Selective Reminding Test delayed).

The Beck Inventory score was slightly higher in high-risk subjects but well below the cutoff of 13 for mild depression (mean 7.3 in high risk and 3.2 in low risk; $p=0.05$ ). The lower Block Design score in high-risk subjects, although only marginally significant, appeared interesting at first because of the association with visuospatial intelligence. However, in a direct comparison of Block Design scaled score between risk groups adjusted for age and education, the difference was not significant (high risk 11.4 \pm 3.70 .4 S.E.M., low risk $12.5 \pm 0.5$ S.E.M.; $p$ $=0.08$ ), tempering the potential significance of this finding.

Figs. 1-3 illustrate statistical maps rendered on the common FA target image in Montreal Neurologic Institute (MNI) spatial coordinates, using the contrast: FA high-AD risk less than FA low risk. The reverse contrast yielded only a few scattered voxels. Maps are referenced to a standard human white matter atlas (Mori et al., 2005). The maps demonstrate lower FA in the high-AD risk subjects in: (a) the inferior longitudinal fasciculus of the temporal lobe posteriorly (particularly inferior fronto-occipital fasciculus bilaterally) and cingulum bundle posteriorly on the left (Fig. 1), (b) splenium of the corpus callosum, white matter of the parahippocampal gyrus (left), and right mid cingulum (Fig. 2), and (c) inferior fronto-occipital fasciculus/uncinate fasciculus anteriorly (Fig. 3). Decreased white matter FA in these specific regions is seen in the younger high- $\mathrm{AD}$ risk group of subjects compared to the older low-AD risk subjects, the reverse of the increased FA expected with aging; therefore the difference cannot be attributed to aging. A listing of the center locations of clusters with $\geq 50$ contiguous voxels (at least $0.05 \mathrm{~cm}^{3}$ volume) is shown in Table 2 . These listings provide a concise summary of relatively spatially coherent FA decreases from results in the figures, which were corrected for multiple comparisons at the significance level but did not impose a cluster threshold. Cluster thresholding refers to comparisons based on spatial extent of FA differences. 


\section{Discussion}

The most important finding was a clearly delineated and highly selective decrease in FA in the inferior temporal lobe white matter bilaterally (inferior longitudinal fasciculus/inferior frontooccipital fasciculus). This decrease has a strong linear spatial character suggesting involvement of a specific group of white matter fibers bridging the amygdala/hippocampal head region anteriorly and the ventral visual association areas posteriorly (Fig. 1).

We also found significant FA decreases in the splenium of the corpus callosum, white matter of the cingulum, and fronto-occipital fasciculus anteriorly. Downward curvature of the involved fronto-occipital fasciculus shown in Fig. 3, particularly well seen on the left, is characteristic of this pathway, which parallels the uncinate fasciculus in part of its course. In general our findings were more robust in the temporal and frontal regions than in parietal lobes, and more spatially coherent in the ventral visual association regions, medial temporal lobe, cingulum and frontal lobe than in the parietal lobe or occipital lobe.

Previous studies using DTI in aging and MCI/AD have used several different acquisition and analysis strategies. Studies of normal aging have shown consistently decreased FA and related measures with age, particularly in the frontal white matter with relative sparing of the temporal, parietal and occipital regions (Pfefferbaum and Sullivan, 2003; Furutani et al., 2005; Charlton et al., 2006; Persson et al., 2006a, ${ }^{\text {b; }}$ Sullivan et al., 2006; Grieve et al., 2007; Kochunov et al., 2007; Madden et al., 2007; Taylor et al., 2007; Abe et al., 2008; Hsu et al., 2008; Hugenschmidt et al., 2008). Increases of FA with age have not been reliably observed in any region.

In contrast, only a few studies have reported selective findings in the frontal lobe in MCI or AD (Choi et al., 2005; Sydykova et al., 2007). The remainder have demonstrated more consistent overlapping findings of decreased FA in the cingulum bundle (Fellgiebel et al., 2007; Teipel et al., 2007; Zhang et al., 2007), temporal lobe generally (Xie et al., 2006; Huang et al., 2007; Stahl et al., 2007), parietal lobe and posterior cingulate (Medina et al., 2006; Firbank et al., 2007; Huang et al., 2007; Ding et al., 2008; Rose et al., 2008), medial temporal white matter (Fellgiebel et al., 2007; Huang et al., 2007; Yasmin et al., 2008), inferior temporal lobe, superior and inferior fronto-occipital fasciculi (Xie et al., 2006; Fellgiebel et al., 2007), and splenium of the corpus callosum (Stahl et al., 2007). Findings in MCI tend to overlap with $\mathrm{AD}$, particularly in posterior areas. These studies have differed in that some selected a restricted target region of a priori interest, e.g., the medial temporal white matter or cingulate bundles (Zhang et al., 2007; Ding et al., 2008; Yasmin et al., 2008), others picked several general regions of interest within lobar white matter (Fellgiebel et al., 2004, 2007; Choi et al., 2005; Stahl et al., 2007), whereas still others used a voxel-based technique (Medina et al., 2006; Rose et al., 2006, 2008; Xie et al., 2006; Firbank et al., 2007; Huang et al., 2007; Sydykova et al., 2007; Teipel et al., 2007). For comparison the analysis technique we used in this study is voxel-based, and therefore unbiased with respect to a specific hypothesis regarding where regional FA differences might be found.

It is important to make the distinction between studies described above that examine subjects with symptoms of a clinically diagnosed cognitive disorder, e.g., MCI and AD, and studies of normal persons without symptoms or deficits on psychometric testing who may later develop these conditions. There are many fewer investigations like the present one that examine the latter issue. Brain FA alterations that are associated with the presence of a diagnosed clinical disorder due to an underlying pathology may be assumed to be different in degree, or perhaps kind, from alterations that predict later development of those symptoms. But it is currently unknown whether such findings would predict MCI or AD. This is implied but not demonstrated in studies of at-risk normal persons. The first step in the validation of the current 
findings is to follow these currently (c. 2008) normal study subjects longitudinally to show whether the observed FA alterations are predictive of later clinically diagnosed MCI or AD.

The voxel-based studies have not been entirely consistent in localization of FA decreases in $\mathrm{AD}$, e.g., some find frontal and internal capsule alterations, but the overall pattern across studies suggests alterations in the white matter of the parahippocampal gyrus, inferior longitudinal fasciculus, splenium of the corpus callosum, parietal white matter, and cingulum bundle (Medina et al., 2006; Rose et al., 2006, 2008; Xie et al., 2006; Firbank et al., 2007; Huang et al., 2007; Sydykova et al., 2007; Teipel et al., 2007).

Of particular relevance, a recent study reported reduced FA in the fornix and left frontal WM in a young group of mildly impaired $(\mathrm{CDR}=0.5)$ subjects (mean age $=35$ years old) at risk for early onset-AD by virtue of fully penetrant genetic alterations (Ringman et al., 2007). Only two studies have explored WM integrity differences in cognitively normal subjects at risk for the more common late-onset form of $\mathrm{AD}$ due to possession of an APOE4 allele. Using ROI or SPM techniques, respectively, these studies observed reduced FA in the left parahippocampal WM (Nierenberg et al., 2005), or the splenium of the corpus callosum, and left posterior hippocampus (Persson et al., 2006a,b). Our results show some overlap with findings in these studies, specifically, the findings of decreased FA in the splenium of the corpus callosum and inferomedial temporal lobes. The location of the present FA decreases in presymptomatic carriers of AD overlap with previous FA reductions observed in $\mathrm{AD}$ in the inferior frontooccipital fasciculi (Xie et al., 2006; Fellgiebel et al., 2007), and cingulum bundle (Fellgiebel et al., 2007; Teipel et al., 2007; Zhang et al., 2007). The term presymptomatic in this context is a group characterization and should not be interpreted to mean that each individual subject should be considered presymtpomatic. These findings therefore suggest that AD-related FA declines are already present in groups of individuals at high risk for $\mathrm{AD}$, even when they are thinking, behaving and functioning normally.

The strengths of our study are the confirmation of cognitive normality and test performance equivalence between risk groups using comprehensive psychometric testing of all participants, rigorous exclusion of confounding neurologic disease, reduction of FA variance due to gender and cerebral language representation by exclusive recruitment of right handed women (Hsu et al., 2008), exclusion of potential confounds due to smoking, hypertension,

hypercholesterolemia and estrogen replacement, a consistent imaging protocol across all subjects using a 3T MRI instrument, and use of an advanced, validated and regionally unbiased DTI methodology.

Several cautions should be kept in mind in interpreting our results. Despite equivalent cognitive performance between risk groups, the low-risk subjects were older than the high-AD risk. We used age as a regressor in all our analyses, but statistical adjustments are not perfect. Because FA is known to decrease - not increase - with age, a finding of an FA decrease in the younger high-risk group is the reverse of expectation on an age basis, so we believe the difference in age strengthens rather than weakens our findings. Nonetheless, the difference in age between groups should be noted.

Another more important caveat is in interpretation of results. We can only infer that the alterations we have observed are related to the risk of $\mathrm{AD}$. The study participants were selected to differ in no respect other than $\mathrm{AD}$ risk, but it is possible the groups differed in some other unknown but important way for which we could not adjust or control. Furthermore, we do not know that presence of these white matter alterations is due to underlying $\mathrm{AD}$ pathology, a basic hypothesis of the study, because we have no independent verification of its degree or presence, e.g., by autopsy or brain biopsy. 
The issue of the functional significance of the FA declines in presymptomatic groups also needs further investigation. This is a tricky issue because presymptomatic high-risk subjects display cognitive function that is, by definition, comparable to that of low-risk subjects. However, there is accumulating evidence that FA is of functional significance in young healthy subjects (Madden et al., 2004; Tuch et al., 2005; Gold et al., 2007), and that age-related FA declines contribute to some cognitive performance declines (Madden et al., 2004; Gold et al., 2008). The view that FA declines are functionally relevant is also in-line with our previous finding of decreased ventral temporal activation in asymptomatic high-AD risk women in an fMRI naming task (Smith et al., 1999). The fMRI under-recruitments in this previous study of subjects were in regions adjacent to the postero-inferior FA alterations observed in the present study. Although both studies were performed using similar subjects selected by the same criteria, the specific individuals studied were different.

Finally, like other studies of presymptomatic participants, we cannot yet claim a role for altered FA in predicting AD because the participants have not been followed long enough to observe even the earliest symptoms for validation. Longitudinal follow up of the participants described in this study is in place at our center and should yield relevant results in the future.

In summary this report represents further progress toward the goal of identifying the brain at risk of AD using DTI. We have identified restricted regions of the inferomedial temporal lobes bilaterally, and other regions, that demonstrate decreased FA in normal high-AD risk subjects, that may in future serve as a biomarker for the presence of asymptomatic AD pathology. What we have observed here are specific regions of decreased FA in high-AD risk subjects. The next step in validating this work is to follow subjects longitudinally to show whether the observed changes are truly predictive of $\mathrm{MCI}$ or $\mathrm{AD}$, and with what accuracy. Therefore, this method is promising but needs further study before it could be used for that purpose in trials of preventive agents in $\mathrm{AD}$.

\section{Supplementary Material}

Refer to Web version on PubMed Central for supplementary material.

\section{Acknowledgments}

Acknowledgements

This study was supported by NINDS Grant R01 NS-36660. We thank Agnes Bognar, R.T., Kim Wilson, B.A., LuAnn Hamon, B.A., Dorothy Ross, B.A., and Barbara Martin, B.A. for their invaluable assistance in recruiting, scanning and testing the participants, and for treating them professionally and so well.

\section{References}

Abe O, Yamasue H, Aoki S, Suga M, Yamada H, Kasai K, Masutani Y, Kato N, Kato N, Ohtomo K. Aging in the CNS: comparison of gray/white matter volume and diffusion tensor data. Neurobiol. Aging 2008;29:102-116. [PubMed: 17023094]

Bennett DA, Schneider JA, Arvanitakis Z, Kelly JF, Aggarwal NT, Shah RC, Wilson RS. Neuropathology of older persons without cognitive impairment from two community-based studies. Neurology 2006;66:1837-1844. [PubMed: 16801647]

Bookstein FL. "Voxel-based morphometry" should not be used with imperfectly registered images. Neuroimage 2001;14:1454-1462. [PubMed: 11707101]

Braak H, Braak E. Frequency of stages of Alzheimer-related lesions of different age categories. Neurobiol. Aging 1997;18:351-357. [PubMed: 9330961]

Charlton RA, Barrick TR, McIntyre DJ, Shen Y, O'Sullivan M, Howe FA, Clark CA, Morris RG, Markus HS. White matter damage on diffusion tensor imaging correlates with age-related cognitive decline. Neurology 2006;66:217-222. [PubMed: 16434657] 
Choi SJ, Lim KO, Monteiro I, Reisberg B. Diffusion tensor imaging of frontal white matter microstructure in early Alzheimer's disease: a preliminary study. J. Geriatr. Psychiatry Neurol 2005;18:12-19. [PubMed: 15681623]

Ding B, Chen KM, Ling HW, Zhang H, Chai WM, Li X, Wang T. Diffusion tensor imaging correlates with proton magnetic resonance spectroscopy in posterior cingulate region of patients with Alzheimer's disease. Dement. Geriatr. Cogn. Disord 2008;25:218-225. [PubMed: 18219201]

Englund E, Brun A, Alling C. White matter changes in dementia of Alzheimer's type. Biochemical and neuropathological correlates. Brain 1988;111(Part 6):1425-1439. [PubMed: 3208064]

Fellgiebel A, Schermuly I, Gerhard A, Keller I, Albrecht J, Weibrich C, Muller MJ, Stoeter P. Functional relevant loss of long association fibre tracts integrity in early Alzheimer's disease. Neuropsychologia. 2007

Fellgiebel A, Wille P, Muller MJ, Winterer G, Scheurich A, Vucurevic G, Schmidt LG, Stoeter P. Ultrastructural hippocampal and white matter alterations in mild cognitive impairment: a diffusion tensor imaging study. Dement. Geriatr. Cogn. Disord 2004;18:101-108. [PubMed: 15087585]

Firbank MJ, Blamire AM, Krishnan MS, Teodorczuk A, English P, Gholkar A, Harrison R, O'Brien JT. Atrophy is associated with posterior cingulate white matter disruption in dementia with Lewy bodies and Alzheimer's disease. Neuroimage 2007;36:1-7. [PubMed: 17412610]

Furutani K, Harada M, Minato M, Morita N, Nishitani H. Regional changes of fractional anisotropy with normal aging using statistical parametric mapping (SPM). J. Med. Invest 2005;52:186-190. [PubMed: 16167537]

Gold BT, Powell DK, Xuan L, Jiang Y, Hardy PA. Speed of lexical decision correlates with diffusion anisotropy in left parietal and frontal white matter: evidence from diffusion tensor imaging. Neuropsychologia 2007;45:2439-2446. [PubMed: 17509627]

Gold BT, Powell DK, Xuan L, Jicha GA, Smith CD. Agerelated slowing of task switching is associated with decreased integrity of frontoparietal white matter. Neurobiol. Aging. 2008 [Epub ahead of print].

Grieve SM, Williams LM, Paul RH, Clark CR, Gordon E. Cognitive aging, executive function, and fractional anisotropy: a diffusion tensor MR imaging study. Am. J. Neuroradiol 2007;28:226-235. [PubMed: 17296985]

Hsu JL, Leemans A, Bai CH, Lee CH, Tsai YF, Chiu HC, Chen WH. Gender differences and age-related white matter changes of the human brain: a diffusion tensor imaging study. Neuroimage 2008;39:566-577. [PubMed: 17951075]

Huang J, Friedland RP, Auchus AP. Diffusion tensor imaging of normal-appearing white matter in mild cognitive impairment and early Alzheimer disease: preliminary evidence of axonal degeneration in the temporal lobe. Am. J. Neuroradiol 2007;28:1943-1948. [PubMed: 17905894]

Hugenschmidt CE, Peiffer AM, Kraft RA, Casanova R, Deibler AR, Burdette JH, Maldjian JA, Laurienti PJ. Relating imaging indices of white matter integrity and volume in healthy older adults. Cereb Cortex 2008;18:433-442. [PubMed: 17575289]

Jones DK, Symms MR, Cercignani M, Howard RJ. The effect of filter size on VBM analyses of DT-MRI data. Neuroimage 2005;26:546-554. [PubMed: 15907311]

Kochunov P, Thompson PM, Lancaster JL, Bartzokis G, Smith S, Coyle T, Royall DR, Laird A, Fox PT. Relationship between white matter fractional anisotropy and other indices of cerebral health in normal aging: tract-based spatial statistics study of aging. Neuroimage 2007;35:478-487. [PubMed: 17292629]

Madden DJ, Spaniol J, Whiting WL, Bucur B, Provenzale JM, Cabeza R, White LE, Huettel SA. Adult age differences in the functional neuroanatomy of visual attention: a combined fMRI and DTI study. Neurobiol. Aging 2007;28:459-476. [PubMed: 16500004]

Madden DJ, Whiting WL, Huettel SA, White LE, MacFall JR, Provenzale JM. Diffusion tensor imaging of adult age differences in cerebral white matter: relation to response time. Neuroimage 2004;21:1174-1181. [PubMed: 15006684]

Medina D, DeToledo-Morrell L, Urresta F, Gabrieli JD, Moseley M, Fleischman D, Bennett DA, Leurgans S, Turner DA, Stebbins GT. White matter changes in mild cognitive impairment and AD: a diffusion tensor imaging study. Neurobiol. Aging 2006;27:663-672. [PubMed: 16005548]

Mori, S.; Wakana, S.; Nagae-Poetscher, LM.; van Zijl, PCM. MRI Atlas of Human White Matter. Elsevier; Amsterdam: 2005. 
Nierenberg J, Pomara N, Hoptman MJ, Sidtis JJ, Ardekani BA, Lim KO. Abnormal white matter integrity in healthy apolipoprotein E epsilon4 carriers. Neuroreport 2005;16:1369-1372. [PubMed: 16056141]

Persson J, Lind J, Larsson A, Ingvar M, Cruts M, Van Broeckhoven C, Adolfsson R, Nilsson LG, Nyberg L. Altered brain white matter integrity in healthy carriers of the APOE epsilon4 allele: a risk for AD? Neurology 2006a;66:1029-1033. [PubMed: 16606914]

Persson J, Nyberg L, Lind J, Larsson A, Nilsson LG, Ingvar M, Buckner RL. Structure-function correlates of cognitive decline in aging. Cereb Cortex 2006b;16:907-915. [PubMed: 16162855]

Pfefferbaum A, Sullivan EV. Increased brain white matter diffusivity in normal adult aging: relationship to anisotropy and partial voluming. Magn. Reson. Med 2003;49:953-961. [PubMed: 12704779]

Ringman JM, O'Neill J, Geschwind D, Medina L, Apostolova LG, Rodriguez Y, Schaffer B, Varpetian A, Tseng B, Ortiz F, Fitten J, Cummings JL, Bartzokis G. Diffusion tensor imaging in preclinical and presymptomatic carriers of familial Alzheimer's disease mutations. Brain 2007;130:1767-1776. [PubMed: 17522104]

Rose SE, Janke AL, Chalk JB. Gray and white matter changes in Alzheimer's disease: a diffusion tensor imaging study. J. Magn. Reson. Imaging 2008;27:20-26. [PubMed: 18050329]

Rose SE, McMahon KL, Janke AL, O'Dowd B, de Zubicaray G, Strudwick MW, Chalk JB. Diffusion indices on magnetic resonance imaging and neuropsychological performance in amnestic mild cognitive impairment. J. Neurol. Neurosurg. Psychiatry 2006;77:1122-1128. [PubMed: 16754694]

Rueckert D, Sonoda LI, Hayes C, Hill DL, Leach MO, Hawkes DJ. Nonrigid registration using free-form deformations: application to breast MR images. IEEE Trans. Med. Imaging 1999;18:712-721. [PubMed: 10534053]

Schneider JA, Arvanitakis Z, Bang W, Bennett DA. Mixed brain pathologies account for most dementia cases in community-dwelling older persons. Neurology 2007;69:2197-2204. [PubMed: 17568013]

Smith CD. Mild cognitive impairment is too late: the case for presymtomatic detection and treatment of Alzheimer's disease. Cogn. Sci 2007;3:127-177.

Smith CD, Andersen AH, Kryscio RJ, Schmitt FA, Kindy MS, Blonder LX, Avison MJ. Altered brain activation in cognitively intact individuals at high risk for Alzheimer's disease. Neurology 1999;53:1391-1396. [PubMed: 10534240]

Smith CD, Andersen AH, Kryscio RJ, Schmitt FA, Kindy MS, Blonder LX, Avison MJ. Women at risk for AD show increased parietal activation during a fluency task. Neurology 2002;58:1197-1202. [PubMed: 11971086]

Smith SM, Jenkinson M, Johansen-Berg H, Rueckert D, Nichols TE, Mackay CE, Watkins KE, Ciccarelli O, Cader MZ, Matthews PM, Behrens TE. Tract-based spatial statistics: voxelwise analysis of multisubject diffusion data. Neuroimage 2006;31:1487-1505. [PubMed: 16624579]

Stahl R, Dietrich O, Teipel SJ, Hampel H, Reiser MF, Schoenberg SO. White matter damage in Alzheimer disease and mild cognitive impairment: assessment with diffusion-tensor MR imaging and parallel imaging techniques. Radiology 2007;243:483-492. [PubMed: 17456872]

Sullivan EV, Adalsteinsson E, Pfefferbaum A. Selective age-related degradation of anterior callosal fiber bundles quantified in vivo with fiber tracking. Cereb Cortex 2006;16:1030-1039. [PubMed: 16207932]

Sydykova D, Stahl R, Dietrich O, Ewers M, Reiser MF, Schoenberg SO, Moller HJ, Hampel H, Teipel SJ. Fiber connections between the cerebral cortex and the corpus callosum in Alzheimer's disease: a diffusion tensor imaging and voxel-based morphometry study. Cereb Cortex 2007;17:2276-2282. [PubMed: 17164468]

Talairach, J.; Tournoux, P. Co-Planar Stereotaxic Atlas of the Human Brain. Thieme Medical Publishers; New York: 1988.

Taylor WD, Bae JN, MacFall JR, Payne ME, Provenzale JM, Steffens DC, Krishnan KR. Widespread effects of hyperintense lesions on cerebral white matter structure. Am. J. Roentgenol 2007;188:16951704. [PubMed: 17515396]

Teipel SJ, Stahl R, Dietrich O, Schoenberg SO, Perneczky R, Bokde AL, Reiser MF, Moller HJ, Hampel H. Multivariate network analysis of fiber tract integrity in Alzheimer's disease. Neuroimage 2007;34:985-995. [PubMed: 17166745] 
Tuch DS, Salat DH, Wisco JJ, Zaleta AK, Hevelone ND, Rosas HD. Choice reaction time performance correlates with diffusion anisotropy in white matter pathways supporting visuospatial attention. Proc. Natl. Acad. Sci. U.S.A 2005;102:12212-12217. [PubMed: 16103359]

Xie S, Xiao JX, Gong GL, Zang YF, Wang YH, Wu HK, Jiang XX. Voxel-based detection of white matter abnormalities in mild Alzheimer disease. Neurology 2006;66:1845-1849. [PubMed: 16801648]

Yasmin H, Nakata Y, Aoki S, Abe O, Sato N, Nemoto K, Arima K, Furuta N, Uno M, Hirai S, Masutani Y, Ohtomo K. Diffusion abnormalities of the uncinate fasciculus in Alzheimer's disease: diffusion tensor tract-specific analysis using a new method to measure the core of the tract. Neuroradiology. 2008

Zhang Y, Schuff N, Jahng GH, Bayne W, Mori S, Schad L, Mueller S, Du AT, Kramer JH, Yaffe K, Chui H, Jagust WJ, Miller BL, Weiner MW. Diffusion tensor imaging of cingulum fibers in mild cognitive impairment and Alzheimer disease. Neurology 2007;68:13-19. [PubMed: 17200485] 


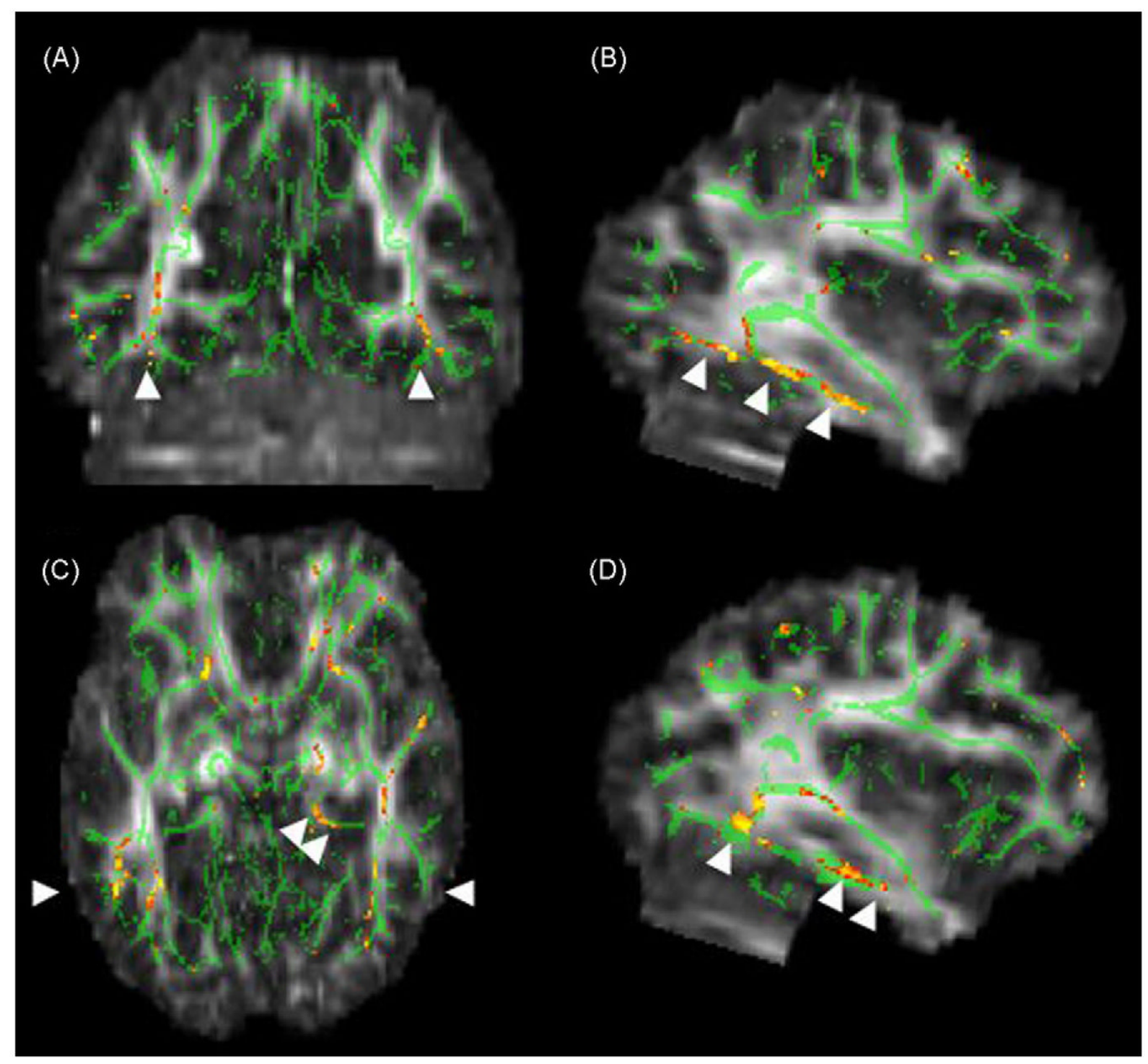

Fig. 1.

Map showing inferotemporal regions of decreased FA in normal, high-AD risk subjects. The anatomic underlay used for illustration is the MNI-space registered target FA image. The registered average FA skeleton is represented in green. Warmer colors on red-orange scale indicate higher $t$-values for FA decrease at each voxel $\left(\mathrm{FA}_{\text {high risk }}<\mathrm{FA}_{\text {low risk }}\right)$. Arrowheads in (A) mark the sagittal slice locations on the right (B) and on the left (D). Coronal slice level in $\mathrm{A}$ is indicated by single arrowheads in (C). The lineal patterns of FA decrease indicated by the arrowheads in (B) and (D) correspond to the inferior longitudinal fasciculus/inferior frontooccipital fasciculus (Mori et al., 2005). Double arrowheads in (C) point to the posterior cingulum bundle on the left. Note that decreased FA adheres only to white matter regions. 

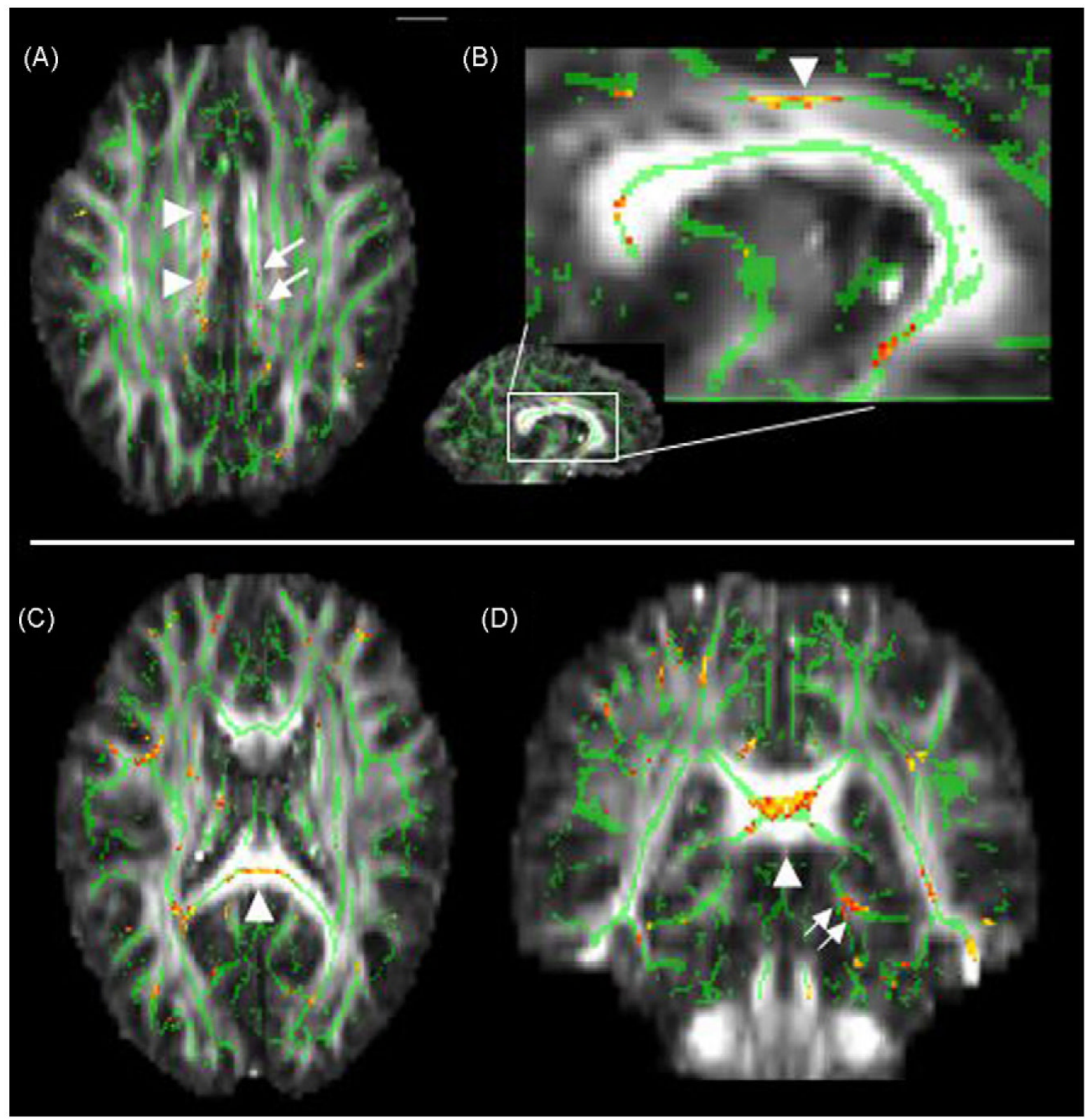

Fig. 2.

Decreased FA in the right mid-cingulum bundle and splenium in normal, high-AD risk subjects. Display conventions are given in Fig. 1 legend. Top row: Arrowheads in (A) and (B) indicate the mid-portion of the cingulum on the right demonstrating decreased FA. A magnified view of the region indicated by a box in the mid-sagittal slice is illustrated in B. Arrows in (A) denotes symmetric cingulate areas with decreased FA on the left, but this region does not have the lineal spatial coherence of the right-sided counterpart. Bottom row: Arrowheads in (C) and (D) mark the region of decreased FA in the splenium of the corpus callosum. Double arrows in (D) indicate decreased FA in the posterior cingulate bundle on the left. 


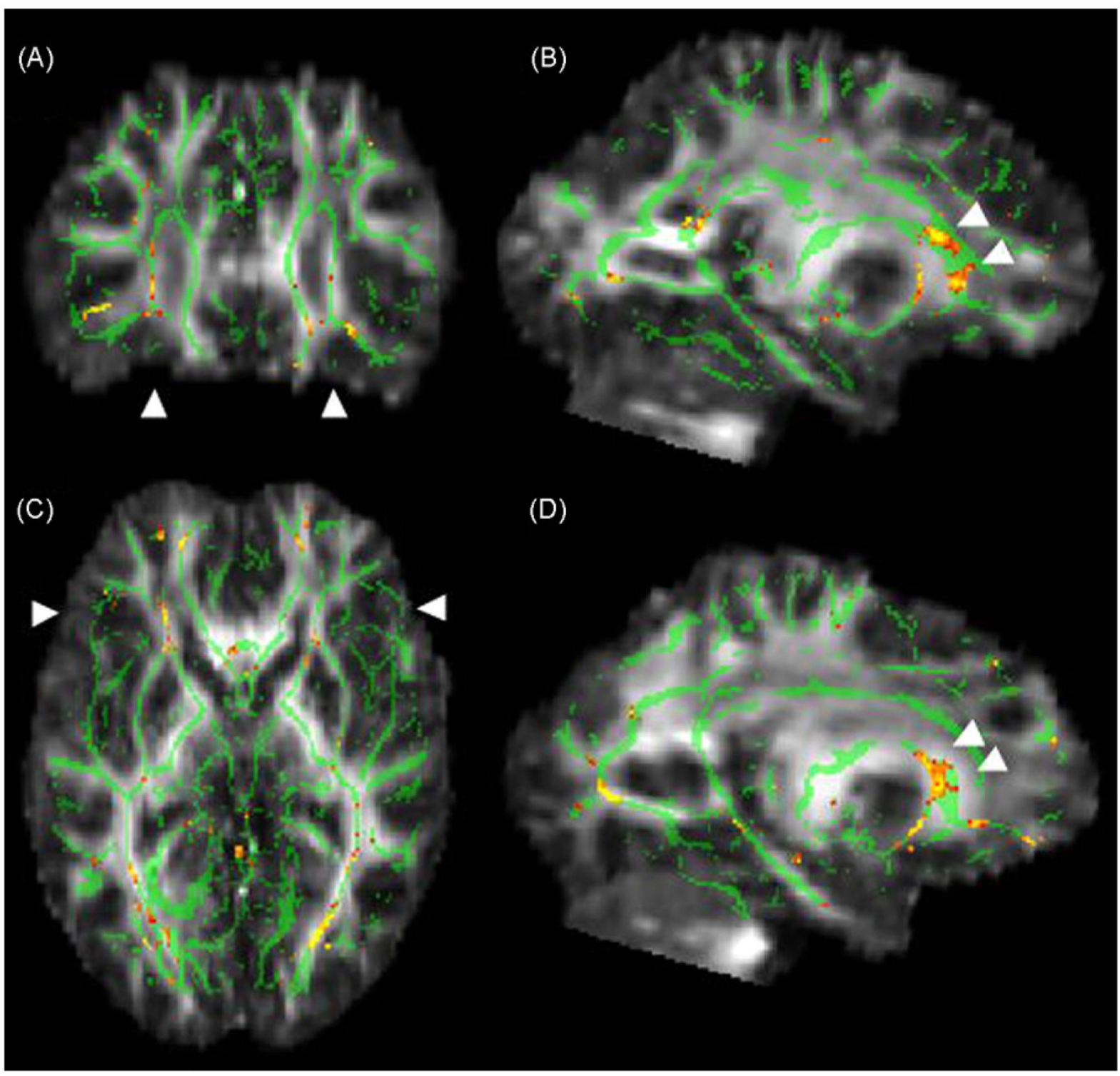

Fig. 3.

Map showing anterior regions of decreased FA in normal, high-AD risk subjects. Display conventions are given in Fig. 1 legend. Arrowheads in A mark the sagittal slice locations on the right (B) and on the left (D). Coronal slice level in A is indicated by single arrowheads in (C). The coherent patterns of FA decrease indicated by the arrows in (B) and (D) correspond to the inferior fronto-occipital fasciculus(Mori et al., 2005). Downward curvature of the FA decrease region, best seen on the left in (B), is typical of this tract. 


\section{Table 1A}

Age and education of study participants

\begin{tabular}{lcll}
\hline Group & N & Age, years & Education, years \\
\hline High risk & 42 & $57.7 \pm 1.1$ & $15.7 \pm 0.4$ \\
Low risk & 23 & $68.1^{* *} \pm 1.5$ & $15.9 \pm 0.6$ \\
$p$-Value & - & $<0.0001$ & 0.73 \\
\hline
\end{tabular}

Standard Least Squares analysis using age and education as response variables and risk as regressor (Least square means \pm S.E.M.). 


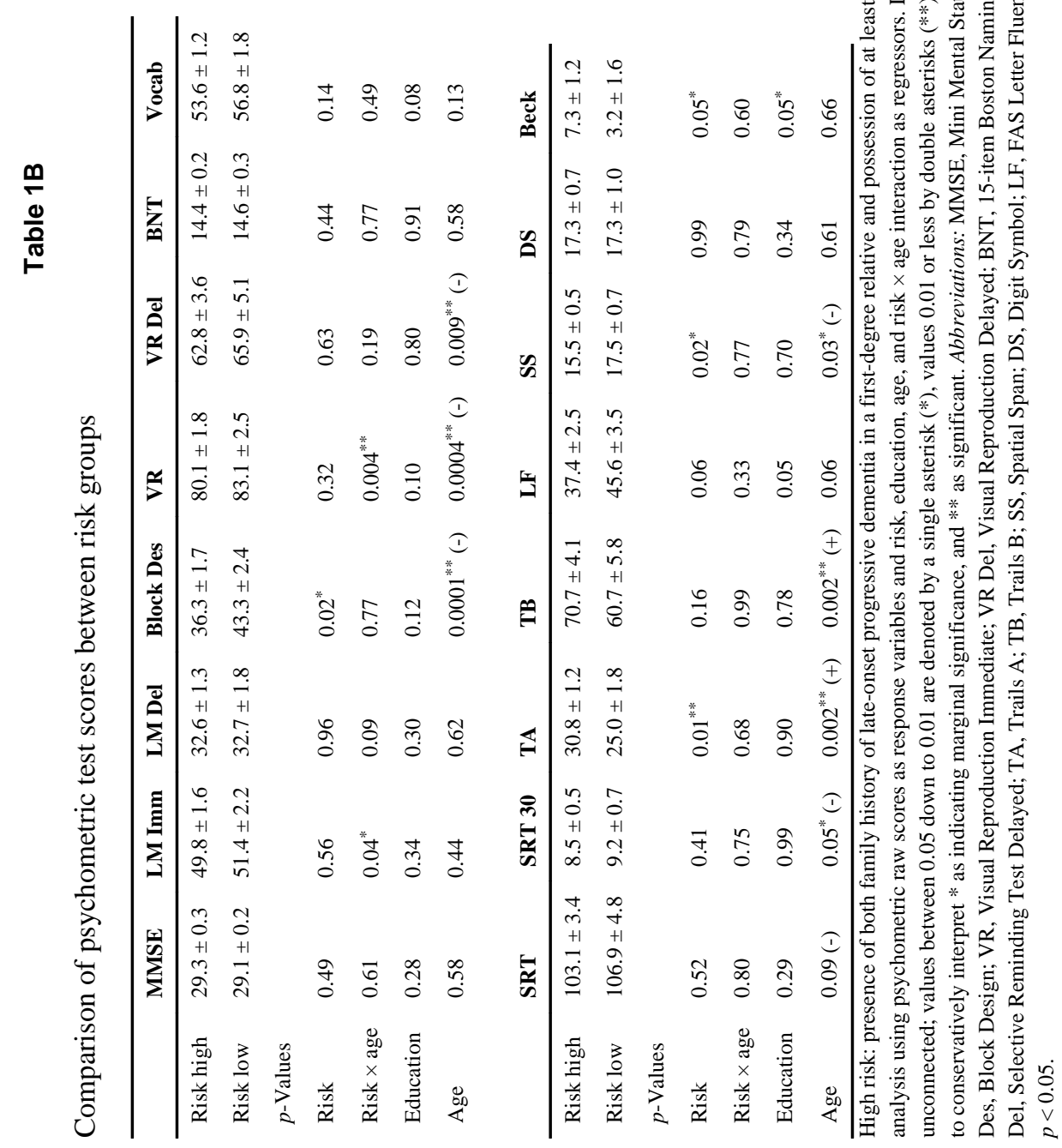


Table 2

Locations of voxels with decreased FA in high-AD risk participants

\begin{tabular}{lll}
\hline Location & Tract & Nearest BA \\
\hline$-27,-77,2$ & Inferior fronto-occipital fasciculus L (posterior) & 19 \\
$25,-78,2$ & Inferior fronto-occipital fasciculus R (posterior) & 19 \\
$-38,-63,-8$ & Inferior fronto-occipital fasciculus L (posterior) & 19,37 \\
$39,-64,-8$ & Inferior fronto-occipital fasciculus R (posterior) & 19,37 \\
$-37,-55,0$ & Inferior longitudinal fasciculus L (posterior) & 37 \\
$36,-59,5$ & Inferior longitudinal fasciculus R (posterior) & 37 \\
$-19,-36,-7$ & Cingulum bundle L (posterior) & 27,30 \\
$7,-8,32$ & Cingulum bundle R (mid) & 23,24 \\
$-42,-25,-6$ & Inferior fronto-occipital fasciculus L (posterior) & 21 \\
$-15,2,56$ & Cortico-pontine tract & 6 \\
$-24,23,8$ & Inferior fronto-occipital fasciculus L (anterior) & - \\
$26,28,2$ & Inferior fronto-occipital fasciculus L (anterior) & - \\
$0,-36,21$ & Splenium, corpus callosum & - \\
$-4,3,-10$ & Subcallosal white matter & 25 \\
$4,3,-10$ & Subcallosal white matter & 25 \\
\hline
\end{tabular}

Locations are given in Talairach coordinates in the first Column (Talairach and Tournoux, 1988, 1027), the corresponding tract associated with this anatomic location in the second Column (Mori et al., 2005 \#67), and the nearest neighboring Brodmann cortical area (BA) to the location in the third Column. 